\title{
Factors Associated With Discharge Destination in Advanced Cancer Patients With Bone Metastasis in a Japanese Hospital
}

\author{
Katsuhiro Hayashi, $\mathrm{MD}, \mathrm{PhD}^{1}$, Tetsutaro Yahata, $\mathrm{MD}, \mathrm{PhD}^{2}$, Ryota Muramoto, $\mathrm{RPT}^{2}$, \\ Norio Yamamoto, $\mathrm{MD}, \mathrm{PhD}^{1}$, Akihiko Takeuchi, $\mathrm{MD}, \mathrm{PhD}^{1}$, Shinji Miwa, $\mathrm{MD}, \mathrm{PhD}^{1}$, \\ Takashi Higuchi, $\mathrm{MD}^{1}$, Kensaku Abe, $\mathrm{MD}^{1}$, Yuta Taniguchi, $\mathrm{MD}^{1}$, Hisaki Aiba, $\mathrm{MD}^{1}$, \\ Yoshihiro Araki, $\mathrm{MD}^{1}$, Hiroyuki Tsuchiya, $\mathrm{MD}, \mathrm{PhD}^{1}$
}

\begin{abstract}
${ }^{1}$ Department of Orthopaedic Surgery, Graduate School of Medical Sciences, Kanazawa University, Kanazawa; ${ }^{2}$ Section of Physical and Rehabilitation Medicine, Central Medical Facilities, Kanazawa University Hospital, Kanazawa, Japan
\end{abstract}

Objective To analyze patient characteristics of cancer rehabilitation and outcomes at our hospital.

Methods This retrospective study analyzed 580 patients, who underwent cancer rehabilitation at our hospital and rehabilitation outcome after therapy were investigated. The relationship between the initial Barthel index and discharge outcomes was investigated, with a special focus on cancer patients with bone metastasis. The Barthel index and performance status (Eastern Cooperative Oncology Group) before and after rehabilitation were analyzed, and threshold value of home discharge was calculated from a receiver operating characteristic curve (ROC). General criteria for home discharge from our hospital included independence in performing basic activities of daily living such as bathing, feeding, and toileting or availability of home support from a family member/caregiver.

Results The outcomes after rehabilitation among all the patients were as follows: discharge home 59\%, death $13 \%$, and others $27 \%$. Statistical differences were observed between the initial and final values of the Barthel index in patients with bone metastasis, who could be discharged home ( $\mathrm{p}=0.012)$. ROC analysis of the initial Barthel index for predicting home discharge revealed a threshold value of 60 , sensitivity of 0.76 , and specificity of 0.72 .

Conclusion The patients with bone metastasis had a lower rate of home discharge and a higher rate of mortality than all the study patients who underwent cancer rehabilitation at our hospital. It is proposed that at the time of initiation of rehabilitation for patients with bone metastasis, an initial Barthel index lower than 60 might predict a worse outcome than home discharge.

Keywords Neoplasms, Rehabilitation, Treatment outcome

Received June 19, 2017; Accepted August 21, 2017

Corresponding author: Katsuhiro Hayashi

Department of Orthopaedic Surgery, Graduate School of Medical Sciences, Kanazawa University, Takaramachi 13-1, Kanazawa, Japan. Tel: +81-76265-2374, Fax: +81-76-234-4261, E-mail: khayashi830@gmail.com

ORCID: Katsuhiro Hayashi (http://orcid.org/0000-0001-8665-2154); Tetsutaro Yahata (http://orcid.org/0000-0001-8782-953X); Ryota Muramoto (http://orcid.org/0000-0001-6007-2450); Norio Yamamoto (http://orcid.org/0000-0002-7250-625X); Akihiko Takeuchi (http://orcid.org/0000-00024071-5620); Shinji Miwa (http://orcid.org/0000-0002-5962-8287); Takashi Higuchi (http://orcid.org/0000-0003-3281-4669); Kensaku Abe (http://orcid. org/0000-0002-7405-9019); Yuta Taniguchi (http://orcid.org/0000-0002-4322-6566); Hisaki Aiba (http://orcid.org/0000-0002-3438-9814); Yoshihiro Araki (http://orcid.org/0000-0001-5783-109X); Hiroyuki Tsuchiya (http://orcid.org/0000-0003-0730-7921).

(c) This is an open-access article distributed under the terms of the Creative Commons Attribution Non-Commercial License (http://creativecommons.org/ licenses/by-nc/4.0) which permits unrestricted noncommercial use, distribution, and reproduction in any medium, provided the original work is properly cited. Copyright $\odot 2018$ by Korean Academy of Rehabilitation Medicine 


\section{INTRODUCTION}

Modern medical technology, advances in cancer diagnosis, surgery, and drugs, along with the implementation of cancer boards and multidisciplinary therapy have resulted in an increase in survival rate among cancer patients. The advancements in technology has not only resulted in an increase in the number of cured patients but also an increase in the number of patients living with cancer. Every cancer patient has to cope with minor or serious impairments during hospitalization or as a discharged patient due to cancer progression or adverse effects associated with treatment. Metastases to bone, brain, lung, or other organs are closely related to a patient's activities of daily living. For instance, patients with bone metastasis are at increased risk of skeletal-related events (SREs) such as pain, fracture, or paralysis caused by neural compression. SREs can greatly impede a patient's ability to be physically active, which might lead to shorter survival [1].

Rehabilitation can help maintain and improve the quality of life of cancer patients throughout the course of the disease, from early to advanced-stage cancer. Comprehensive reports on the need for rehabilitation were first published in 1978 [2]. They stated that rehabilitation care can improvise many disease-related problems. In 1982, a report by Harvey et al. [3] based on literature search and survey analysis provided supportive remarks on the implementation of a team approach for the rehabilitation of cancer patients. Dietz [4] classified cancer rehabilitation into four categories as preventive, restorative, supportive, and palliative rehabilitation. Rehabilitation has been reported to be effective for each stage of cancer treatment, starting from physical rehabilitation during the acute stage of treatment to the rehabilitation program addressing physical and psychological problems during the terminal stage. A retrospective study by Miyashita et al. [5] reported on the discharge of cancer patients from a palliative care unit in Japan and the related factors. The study found several factors associated with discharge such as performance status and problems with oral intake. However, the existing reports on discharge outcome of patients with bone metastasis lack strong evidence.

Since 1981, cancer has been considered as the leading cause of death in Japan. The Cancer Control Act was approved in 2006, and the law was implemented in 2007.
The basic concepts of the law include promotion of cancer research, equalization of cancer medical service, and development of cancer medical services to satisfy patients. The revised version of the Basic Plan to Promote Cancer Control Programs now includes specific goals for cancer treatment, such as rehabilitation. The Japanese government implements its cancer control policy in cooperation with local communities; the public, including the cancer patients; medical facilities; health insurance companies; academic associations; patient groups; and the mass media. Interest in cancer rehabilitation has increased ever since the cancer patient rehabilitation fee was newly established in the 2010 revision of medical care service fees [6]. However, substantiating the need for rehabilitation services has been adequately addressed by cancer professionals and caregivers remain difficult. Additional research on the benefit of rehabilitation is needed, because of the scarcity in the high-quality evidence on cancer rehabilitation in the literature. Guidelines on cancer rehabilitation were published by the Japanese Association of Rehabilitation Medicine in 2013 and were based on evidence-based published data. The guidelines for the medical staff strongly support cancer rehabilitation.

In this study, we analyzed collected data on cancer rehabilitation at our hospital, with a special focus on cancer patients with bone metastasis. Bone metastasis is a serious condition that can have a negative impact on patients with advanced cancer. Patients with bone metastasis may experience limitations in the activities of daily living especially when the disease is accompanied by SREs. Advancements in modern medical technologies have not only resulted in an increase in the number of cured patients but also an increase in the number of patients living with cancer. Management of bone metastasis is one of the keys of cancer treatment to maintain the patient's activity. The aim of the study was to analyze patient characteristics of cancer rehabilitation and outcomes and to investigate the relationship between the initial Barthel index and discharge outcomes.

\section{MATERIALS AND METHODS}

This retrospective study analyzed 580 patients (361 males and 219 females; mean age, 62.1 years), who underwent cancer rehabilitation at Kanazawa University 
Hospital between 2014 and 2015 (Table 1). Among the 580 patients, 99 ( 51 males and 48 females; mean age, 63.1 years) had bone metastasis. A number of bone metastasis was solitary in 32 cases, 2 sites in 11 cases, 3 sites in 4 cases, 4 sites in 1 case, and 5 and more sites in 51 cases. Weight-bearing site of metastasis was found in 73 cases. Neurological symptoms were seen in 28 cases. All the study patients had the following primary tumors: 80 patients with lung cancer, 68 with leukemia, 54 with lymphoma, 48 with hepatocellular carcinoma, 37 with esophageal cancer, 35 with gastric cancer, and 258 with other cancers. The patients with bone metastasis had the following primary tumors: 32 with lung cancer, 12 with breast cancer, 9 with kidney cancer, 6 with prostate cancer, and 40 with other cancers. The treating departments for all the patients included Hematology for 97, HepatoBiliary-Pancreatic Surgery for 62, Gastroenterological Surgery for 59, and other departments for 362 patients. The treating departments for patients with bone metastasis included Urology for 20, Pulmonary Medicine for 17, Gastrointestinal Medicine for 11, and other depart- ments for 51 patients. Rehabilitation type and outcome after therapy were investigated for all the patients and for the patients with bone metastasis. The Barthel index and performance status (Eastern Cooperative Oncology Group) before and after rehabilitation were analyzed by the Student t-test, and the threshold value of home discharge and area under the curve (AUC) was calculated from a receiver operating characteristic (ROC) curve. General criteria for home discharge from our hospital included independence in performing basic activities of daily living such as bathing, feeding, and toileting or availability of home support from a family member/ caregiver. Most of the cancer patients and medical staff wish for home discharge and it is one of the goals of rehabilitation. However, at this stage, we are not sure about the condition that will predict home discharge in cancer patients.

All the statistical analyses were performed with EZR software (Saitama Medical Center, Jichi Medical University, Saitama, Japan), which is a graphical user interface for R (The R Foundation for Statistical Computing, Vien-

Table 1. Characteristics of cancer patients undergoing rehabilitation

\begin{tabular}{|c|c|c|}
\hline & All patients $(n=580)$ & Patients with bone metastasis $(n=99)$ \\
\hline Age (yr) & $62.1(7-95)$ & $63.2(7-92)$ \\
\hline \multicolumn{3}{|l|}{ Sex } \\
\hline Male & 361 & 51 \\
\hline Female & 219 & 48 \\
\hline \multirow[t]{16}{*}{ Primary tumor } & Lung cancer (80) & Lung cancer (32) \\
\hline & Leukemia (68) & Breast cancer (12) \\
\hline & Lymphoma (54) & Kidney cancer (9) \\
\hline & Hepatocellular carcinoma (48) & Prostate cancer (6) \\
\hline & Esophageal cancer (35) & Gastric cancer (5) \\
\hline & Gastric cancer (37) & Pancreatic cancer (5) \\
\hline & Pancreatic cancer (35) & Childhood cancer (3) \\
\hline & Head and neck cancer (28) & Skin cancer (3) \\
\hline & Colon/rectal cancer (21) & Bladder cancer (3) \\
\hline & Biliary tract cancer (20) & Others (21) \\
\hline & Uterine cancer (17) & \\
\hline & Sarcoma (17) & \\
\hline & Breast cancer (16) & \\
\hline & Kidney cancer (14) & \\
\hline & Prostate cancer (11) & \\
\hline & Others (79) & \\
\hline
\end{tabular}

Values are presented as mean (range). 
na, Austria) [7]. More precisely, EZR software is a modified version of $\mathrm{R}$ commander, which was designed to add statistical functions frequently used in biostatistics. The Institutional Review Board of Kanazawa University Hospital approved this study (IRB No. 2373-1).

\section{RESULTS}

Physical therapy (PT) was performed for all the enrolled 580 patients (100\%), occupational therapy (OT) for $11 \%$, and speech therapy (ST) for $21 \%$ (Table 2). Among the 99 patients with bone metastasis, PT was performed for $99 \%$, OT for $11 \%$, and ST for $29 \%$. The mean duration of rehabilitation was 51.7 and 48.3 days, respectively. The outcomes after rehabilitation among all the patients were as follows: discharge home 59\%, death $13 \%$, and others $27 \%$ (102 cases changed hospital, 8 cases moved to health

Table 2. Details of rehabilitation therapy and outcomes

\begin{tabular}{lcc}
\hline & $\begin{array}{c}\text { All } \\
\text { patients }\end{array}$ & $\begin{array}{c}\text { Patients } \\
\text { with bone } \\
\text { metastasis }\end{array}$ \\
\hline $\begin{array}{l}\text { Therapy } \\
\text { Physical therapy }\end{array}$ & \\
\hline $\begin{array}{l}\text { Occupational therapy } \\
\text { Speech therapy }\end{array}$ & $120(11)$ & $29(11)$ \\
$\begin{array}{l}\text { Mean rehabilitation period (day) } \\
\text { Outcome }\end{array}$ & $55(21)$ & $11(29)$ \\
\hline Home discharge & & 48.3 \\
\hline Death & $345(59)$ & $44(44)$ \\
\hline Others (changing hospital, etc.) & $158(27)$ & $32(32)$ \\
\hline
\end{tabular}

Values are presented as number (\%). services facility, and 48 cases terminated from rehabilitation due to some other reasons). Among patients with bone metastasis, the outcomes after rehabilitation were as follows: discharge home $44 \%$, death $23 \%$, and others $32 \%$, which were worse outcomes compared to the outcomes of other patients. According to the clinical outcome (discharge destination) of the patients with bone metastasis, the average duration of rehabilitation was as follows: discharge home 56.7 days, death 35.1 days, and others 42.9 days, which was not statistically different.

The Barthel indices of patients with bone metastasis before and after rehabilitation were 52.2 and 54.3, respectively. The performance status values of the patients with bone metastasis before and after rehabilitation were 3.1 and 3, respectively. Stratified by the outcome, statis-

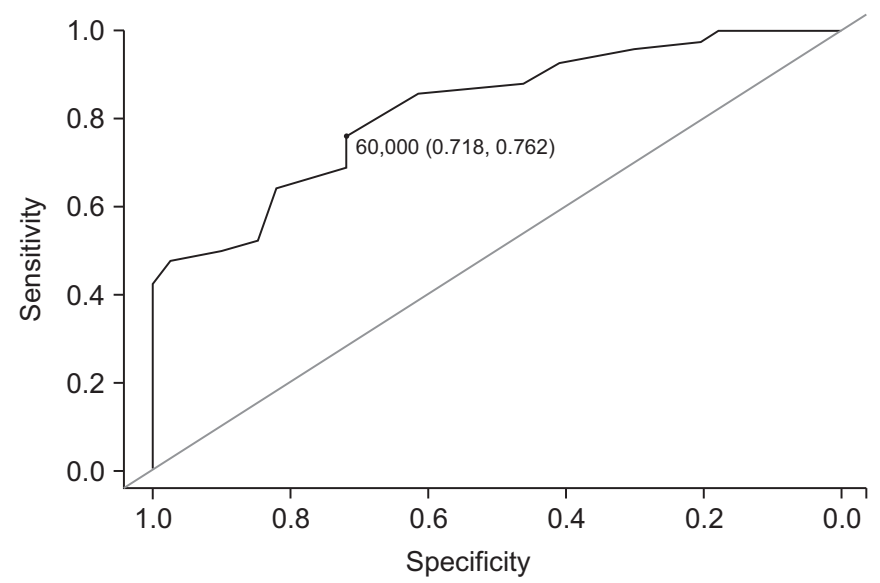

Fig. 1. ROC analysis of initial Barthel index for predicting home discharge. AUC $=0.82$ (95\% confidential interval, $0.73-0.91$ ), threshold value $=60$, sensitivity $=0.76$, specificity $=0.72$. ROC, receiver operating characteristic; AUC, area under the curve.

Table 3. Initial and final scores of Barthel index and performance status in patients with bone metastasis

\begin{tabular}{|c|c|c|c|c|}
\hline & Discharged & Death & Others & All \\
\hline \multicolumn{5}{|c|}{ Barthel index } \\
\hline Initial & $69.4(25-100)$ & $40.9(0-75)$ & $31(0-90)$ & $52.2(0-100)$ \\
\hline Final & $81.7(25-100)$ & $38.2(0-80)$ & $18.8(0-90)$ & $54.3(0-100)$ \\
\hline p-value & $0.012^{*}$ & 0.094 & 0.72 & 0.67 \\
\hline \multicolumn{5}{|c|}{ Performance status } \\
\hline Initial & $2.7(1-4)$ & $3.2(3-4)$ & $3.7(1-4)$ & $3.1(1-4)$ \\
\hline Final & $2.4(1-4)$ & $3.3(3-4)$ & $3.9(1-4)$ & $3(1-4)$ \\
\hline $\mathrm{p}$-value & 0.051 & 0.28 & 0.71 & 0.43 \\
\hline
\end{tabular}

Values are presented as mean (range). ${ }^{*} \mathrm{p}<0.05$. 
tical differences were observed between the initial and final values of the Barthel index of patients who could be discharged home $(\mathrm{p}=0.012)$ (Table 3$)$. ROC analysis of the initial Barthel index for predicting home discharge revealed a threshold value of 60 with a sensitivity of 0.76 and specificity of 0.72 (Fig. 1).

\section{DISCUSSION}

The changes in the cancer rehabilitation service fee for Japanese medical care has provided numerous advantages for patients, including an unlimited period of rehabilitation during admission and more comprehensive physical therapy after surgery for cancer involving various body structures [8]. Cancer patients may need different types of rehabilitation during the early to terminal stage of the disease, and we need to assess the pros and cons of cancer rehabilitation with respect to a range of analyses.

The survey of data from our institution reveals that rehabilitation interventions for all study patients and patients with bone metastasis were performed most frequently for patients with lung cancer. Among patients with bone metastasis, rehabilitation was frequently performed for patients with kidney, breast, or prostate cancers, which are known to metastasize frequently to the bone. The patients with bone metastasis had a lower rate of home discharge and a higher rate of mortality compared to other study patients who underwent cancer rehabilitation. The results might be accounted based on more advanced disease stage of the patients with bone metastasis and by the close relationship between activities of daily living and bone metastasis. The Barthel index of patients with bone metastasis improved after rehabilitation, while their performance scores were slightly worse, but none of the differences were significant. The difference between the Barthel indices before and after rehabilitation of patients who were discharged home was significant. The threshold value of the initial Barthel index of the patients who were discharged home was calculated. The ROC curve revealed moderate accuracy in the AUC and the cut-off Barthel index value was determined to be 60 . It is suggested that at the time of initiation of rehabilitation for patients with bone metastasis, an initial Barthel index lower than 60 might predict a worse outcome than home discharge.

SREs caused due to bone metastasis adversely affect the treatment outcome and are directly related to the activities of daily living. The prediction of pathologic fractures by radiological findings remains a difficult task, although many predictive scores have been published [9]. Rehabilitation for patients with bone metastasis is a challenge because of the special care needed to prevent the occurrence of fractures. However, Bunting and Shea [1] reported a retrospective analysis that stated that the incidence of pathologic fractures of long bones during physical therapy was not very high (1/96), and the advantages of physical therapy were greater than the disadvantages. Ruff et al. [10] performed a randomized controlled trial of physical therapies, including transferring, respiratory training, and urination and defecation training, for patients with spinal metastasis and found that higher proportion of the rehabilitation group had pain relief $(p<0.001)$ and improvement in depression $(p<0.001)$ compared with the control group. Another randomized controlled trial for patients with prostate cancer and bone metastasis revealed that the rehabilitation group obtained better clinical outcomes for physical function and activity without any severe adverse events than the control group [11].

The predictors of discharge outcomes of patients with a variety of conditions such as stroke and spinal cord injury have previously been reported $[12,13]$. A retrospective study by Miyashita et al. [5] concentrated on the discharge of cancer patients from a palliative care unit in Japan and the related factors. They found that the duration of the period from referral to death, performance status, problems with oral intake, the presence of nausea, experience with radiation therapy, and patient's anxiety for the family were independent predictive factors for discharge. Early referral and development of therapies to alleviate symptoms such as nausea, anxiety, and depression are important for increasing the probability of discharge. The existing reports on discharge outcome of patients with bone metastasis do not provide strong evidence on predictive factors. The study on bone metastasis includes various factors, such as the risk of pathologic fracture or spinal cord injury, and a variety of factors associated with each type of primary cancer. It is hypothesized that a prospective study involving patients with bone metastasis will provide information on the types of rehabilitation programs considered to be important, on the methods for reducing the risk of fracture 
during rehabilitation, and on any other factors that will predict or improve discharge outcomes. There are several limitations of this study that deserve consideration. This is a retrospective study and not all the cancer patients underwent cancer rehabilitation in our hospital. The discharge criteria were not clearly defined and influenced by various other factors. The statistical analysis was performed for only patients with bone metastasis, although they were in different situations such as diverse clinical courses of cancer treatment and existence of fracture or paralysis.

\section{CONFLICTS OF INTEREST}

No potential conflict of interest relevant to this article was reported.

\section{REFERENCES}

1. Bunting RW, Shea B. Bone metastasis and rehabilitation. Cancer 2001;92(4 Suppl):1020-8.

2. Lehmann JF, DeLisa JA, Warren CG, deLateur BJ, Bryant PL, Nicholson CG. Cancer rehabilitation: assessment of need, development, and evaluation of a model of care. Arch Phys Med Rehabil 1978;59:410-9.

3. Harvey RF, Jellinek HM, Habeck RV. Cancer rehabilitation: an analysis of 36 program approaches. JAMA 1982;247:2127-31.

4. Dietz JH. Rehabilitation of the cancer patient. Med Clin North Am 1969;53:607-24.

5. Miyashita M, Arai K, Yamada Y, Owada M, Sasahara T, Kawa M, et al. Discharge from a palliative care unit: prevalence and related factors from a retrospective study in Japan. J Palliat Med 2009;12:142-9.

6. Okamura H. Importance of rehabilitation in cancer treatment and palliative medicine. Jpn J Clin Oncol 2011;41:733-8.

7. Kanda Y. Investigation of the freely available easy-touse software 'EZR' for medical statistics. Bone Marrow Transplant 2013;48:452-8.

8. Hasegawa T, Goto N, Matsumoto N, Sasaki Y, Ishiguro T, Kuzuya N, et al. Prevalence of unmet needs and correlated factors in advanced-stage cancer patients receiving rehabilitation. Support Care Cancer 2016;24:4761-7.

9. Mirels H. Metastatic disease in long bones: a proposed scoring system for diagnosing impending pathologic fractures. Clin Orthop Relat Res 1989;(249):256-64.

10. Ruff RL, Ruff SS, Wang X. Persistent benefits of rehabilitation on pain and life quality for nonambulatory patients with spinal epidural metastasis. J Rehabil Res Dev 2007;44:271-8.

11. Cormie P, Newton RU, Spry N, Joseph D, Taaffe DR, Galvao DA. Safety and efficacy of resistance exercise in prostate cancer patients with bone metastases. Prostate Cancer Prostatic Dis 2013;16:328-35.

12. Fortin CD, Voth J, Jaglal SB, Craven BC. Inpatient rehabilitation outcomes in patients with malignant spinal cord compression compared to other non-traumatic spinal cord injury: a population based study. J Spinal Cord Med 2015;38:754-64.

13. Mees M, Klein J, Yperzeele L, Vanacker P, Cras P. Predicting discharge destination after stroke: a systematic review. Clin Neurol Neurosurg 2016;142:15-21. 